\title{
CONCEPT DESIGN AND INTEGRATION ASPECTS OF ITER VERTICAL NEUTRON CAMERA
}

\author{
Luciano Bertalot', Robin Barnsley, Natalia Casal, Jerome Ferreol, Thibaud \\ Giacomin, Vitaly Krasilnikov, Vincent Martin, Stefan Simrock, Alejandro Suarez, \\ Victor Udintsev, Michael Walsh, Nikolay Yukhnov \\ ITER Organization, Route de Vinon sur Verdon, Saint Paul Lez Durance, France \\ E-mail: Luciano.Bertalot@iter.org
}

Vladimir Amosov, Grigorii Nemtcev, Alexander Zvonkov

Institution "PROJECT CENTER ITER”, 1, Akademika Kurchatova sq., Moscow, Russian Federation

E-mail: $\underline{\text {.zvonkov@iterrf.ru }}$

\section{Fernando Mota}

Laboratorio Nacional de Fusión por Confinamiento Magnético - CIEMAT, Madrid, 28040, Spain

E-mail:fernando.mota@ciemat.es

\section{Flavio Lucca, Anna Marin, Fabio Vigano}

L.T.Calcoli SaS, Piazza Prinetti 26/B, 23807 - Merate (LC), Italy

E-mail: lucca@ltcalcoli.it

\section{Hyeon Gon Lee, Sunil Pak}

ITER Korea, Gwahangno113, Yusung-gu, Daejeon 305-333, Korea

E-mail: hglee@nfri.re.kr

The Vertical Neutron Camera (VNC) is a multichannel neutron collimator intended to characterise fusion plasma neutron source. The ITER VNC diagnostic will measure the time-resolved neutron emission profile, the evaluation of the fusion power density and $\alpha$-source emissivity profile, ion temperature profile. Knowledge of the neutron spatial and energy distribution is also important for optimizing fusion burn control and the auxiliary fusion plasma heating systems. The VNC is composed of two fan-shaped collimating structures: Lower and Upper located in different positions of ITER Vacuum Vessel (VV). The plasma is observed vertically by a number of Lines of Sight (LOS). Lower VNC subsystem will be installed into a dedicated support structure (Diagnostic Rack - DR) in Lower Port \#14 and will have 5 collimating channels passing through DR structure, Divertor Central Cassette \#40 body, central blanket's Shielding Block (SB) and First Wall (FW) of $18^{\text {th }}$ row. Upper VNC subsystem will be mounted in the Port Plug (PP) structure (Upper PP \#18). It will consist of two fan-shaped multichannel collimators with overall 6 LOS (3 in each fan). The collimators pass through Diagnostic Shielding Module (DSM) and Diagnostic First Wall (DFW) of UPP\#18. Each VNC Detector Unit has set of neutron detectors providing the required range of sensitivity, as well as temporal and energy resolution. Together with the VNC, other diagnostics systems are installed in the same locations. The integration/interfaces of these diagnostics with other ITER systems play a key role in the VNC engineering design.

First EPs Conference on Plasma Diagnostics - $1^{\text {st }}$ ECPD

14-17 April 2015,

Villa Mondragone, Frascati (Rome), Italy

${ }^{1}$ Speaker

(C) Copyright 2015 ITER Organization. 


\section{Introduction}

This paper reports results achieved at the conceptual design review of the 55B2 Vertical Neutron camera (VNC) development for ITER [1]. VNC is a multichannel neutron collimator intended to characterize fusion plasma neutron source. The VNC diagnostics (together with 55.B1 Radial Neutron Camera) will measure the time resolved neutron emission profile for both DD and DT ITER plasmas, providing the evaluation of the fusion power density, $\alpha$-source density, neutron and $\alpha$-source emissivity profile, ion temperature profile, fusion power and total neutron flux $[2,3,4]$. System requirements for VNC ask for wide plasma coverage (up to $\mathrm{a} / \mathrm{r}=$ 0.85), time resolution $1 \mathrm{~ms}$ for neutron emission profile and $100 \mathrm{~ms}$ for ion temperature profile.

Acquiring fusion power value with high time resolution plays a key role in machine protection. VNC is called as a backup diagnostic system for this measurement. Synergy of VNC data with other neutron diagnostics can reduce error in fusion power measurement.

By using compact neutron spectrometers such as CVD diamond detectors, measurement of the spatial distribution of the ion temperature in the plasma core and determination of fuel ratio is also possible.

The integration aspects between various port-based diagnostics and interfaces with ITER systems (Vacuum, Water cooling, Remote Handling, etc.) play a key role for the VNC engineering design. This paper summarizes the state-of-art of the ITER VNC system at conceptual design phase and the engineering challenges aiming to meet system requirements and to be in compliance with other machine systems.

\section{System Description}

The VNC is composed of two fan-shaped collimating structures: lower and upper subsystems - located in different positions (ports) of ITER Vacuum Vessel (VV). Plasma is observed vertically by a number of Lines of Sight (LOS). The VNC subsystems will be installed in two locations (see fig.1):

1) Lower VNC system will be mounted into a supporting structure (dedicated Diagnostic Rack DR) located inside Lower Port \#14 and will have 6 Detector Units. Five Detector Units will observe the outboard plasma aiming to achieve a wide plasma coverage $\mathrm{a} / \mathrm{r}<0.85$ through the collimating channels and one (the latter one without a collimator) will measure the background. Channels penetrate through DR structure, Divertor Central Cassette (CC) \#40 body, central blanket's Shielding Block (SB) and First Wall (FW) of $18^{\text {th }}$ row. The collimated neutrons will imping the Detector Module (DM) - sealed tight metal box hosting detectors units (each unit consists of two Fission Chambers and two CVD) and having channels for medium/cooling circulation and signal cables tracing;

2) Upper VNC system will be located inside the UPP\#18 structure. It will consist of two fan-shaped multichannel collimator assemblies with overall 6 LOS (3 in each fan) aggregated in Detectors Module. Channels will pass through Diagnostic Shielding Module (DSM), Diagnostic First Wall (DFW) of UPP\#18. All six Detector Units (of design similarly to the LVNC ones) will detect the collimated neutron flux from the inboard plasma region. 


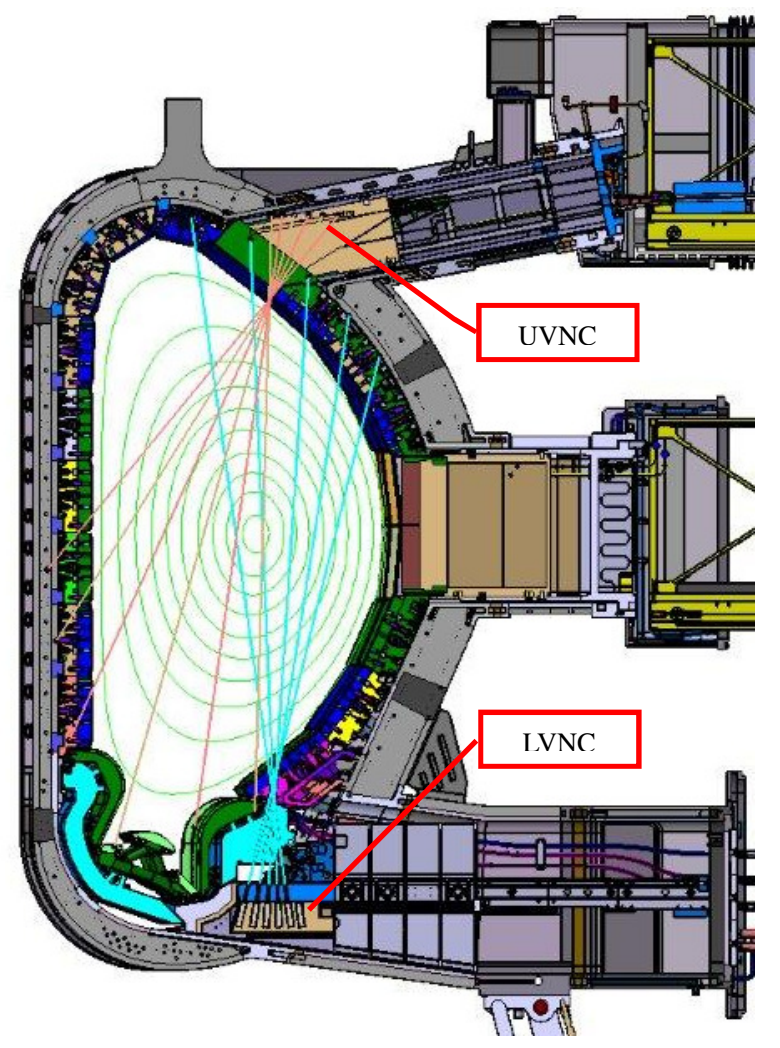

Figure 1 Location of VNC subsystems in the Tokamak Vacuum Vessel (schematic view)

The VNC diagnostics has to withstand high temperatures (up to $250{ }^{0} \mathrm{C}$ during baking phase), magnetic fields up $\sim 1 \mathrm{~T}$ and transient magnetic field of approx. 10T/s. Under these severe load conditions the system requires a strong mechanical support structure, proper shielding and active cooling.

The dedicated Diagnostic Rack for Lower VNC subsystem has additional components which provide more efficient neutron shielding for higher detectors signal-to-background (S/B) ratios. The front part of DR structure has a massive tungsten block ("nose") $150 \mathrm{~mm}$ width and about $880 \mathrm{~mm}$ long, which goes inside the pumping slot of the central Divertor Cassette (fig. 2).

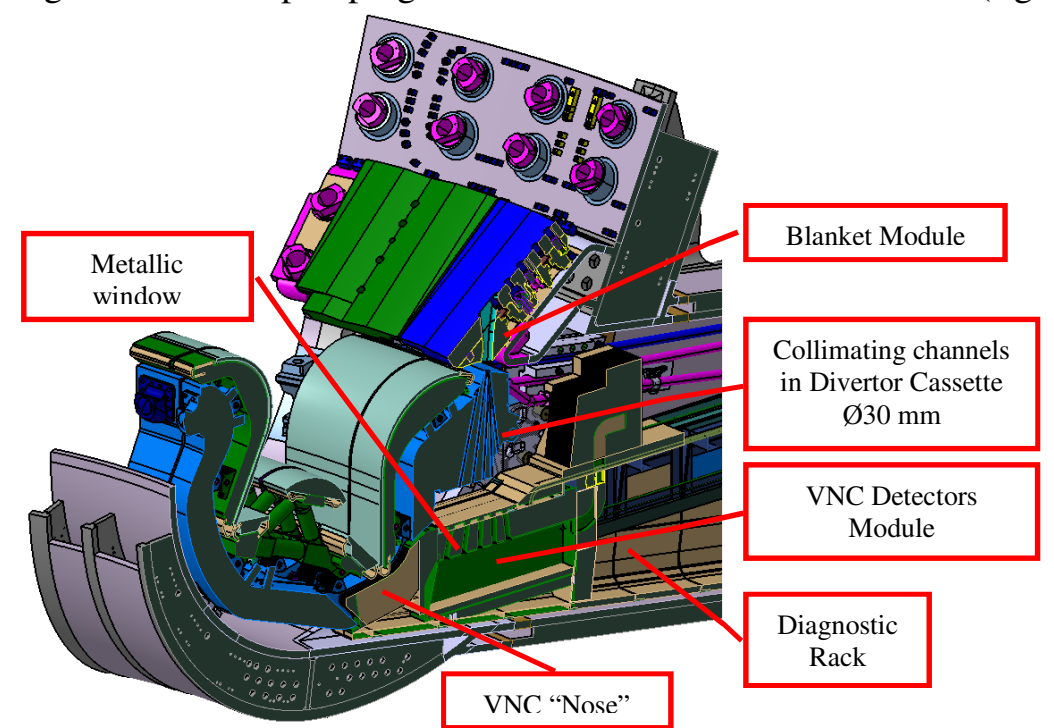

Figure 2 Lower Vertical Neutron Camera in the Lower Port \#14 
Particular care is given to the electrical connections of the Detector Units by using triaxial mineral insulated cables aiming to high noise rejection. Metallic vacuum windows will be installed at the end of each collimator. VNC Detectors Modules are connected to the Service Vacuum System (SVS) to avoid trapped volume and protect torus vacuum from possible leakages of detector filling gas.

\section{VNC detectors calibration}

The front-end electronic of ITER diagnostic systems will be exposed to nuclear radiation. The strategy to calibrate the neutron systems on ITER is described in ref. [5, 6]. Following are the specific calibration requirements for VNC [1]:

a) In-situ calibration of neutron diagnostics is planned before the beginning of the ITER nuclear phase. Neutron sources will be moved in front of VNC entrance to determine the position of collimators and to check the detection functioning. This procedure for VNC has to verify the correct installation and adjustment of the both upper and lower systems.

b) Long term stability and periodic calibrations are needed for VNC detectors during ITER life because their characteristics may vary due to changes of the magnetic field, temperature drift and degradation of sensitivity due to high neutron/gamma fluxes. In the case of Fission Chamber check can be performed using the impurity of alpha sources (like ${ }^{234} \mathrm{U}$ ). Operation of diamond detectors can be controlled with combined alpha, beta and gamma sources. In this case alpha and beta sources are used to provide the energy scale in the high and low ranges, the gamma source allows to evaluate the efficient detector volume.

In the energy range of $100 \mathrm{keV}-10 \mathrm{MeV}$ the dominant process of gamma interaction is the Compton Effect. If one uses relatively thin diamond detector (typical crystal's size is $5 \times 5 \times 0.5 \mathrm{~mm}$ ) it can be assumed that the scattered photon leaves the detector volume without repeated interaction and the detector signal is completely defined by the ionization produced by Compton electrons. For high energy electrons only a portion of their energy is deposited in such detector's volume and goes into formation of electron-ion pairs. Typically the energy response from $14 \mathrm{MeV}$ neutrons will be much higher than gamma response and can be easily discriminated. But if one has built-in relatively low energy gamma and beta sources (for example Cs-137 with photon energy $661.7 \mathrm{keV}$ ) and no neutrons emission in principle one can register these photons quite well. From the response function on gamma radiation the effective volume of the detector can be calculated. This technique is widely used when one works with HpGe detectors. Of course in case of ITER one has to also take into account activation of surrounding materials.

These weak calibration sources will be installed together with the detectors into the VNC detectors modules for permanent tests of the detector parameters and acquisition system fine tuning. 


\section{Performance Assessment}

Performance of VNC system is assessed by means of analysing the S/B ratio, dynamic range of measurement and accuracy in the source emissivity profile reconstruction.

\subsection{Signal-to-background $(\mathrm{S} / \mathrm{B})$ ratio}

Detectors' S/B ratios were determined with the help of neutron transport code MCNP5 v.1.51. The ITER reference model C-lite, v.1 rev. 131031 was used for the calculations. This model consists of a $40^{\circ}$ regular sector, up to the Bioshield, with reflective boundary conditions at the lateral sides of the sector.

ITER plasma scenario II at 500 MW DT fusion power was used as neutron emission source profile for the calculations.

\subsubsection{Upper VNC detectors}

S/B was evaluated for the candidate neutron VNC detectors: Fission Chamber (FC) with ${ }^{238} \mathrm{U}$ as fissile material and diamond detector. Signal and background are computed in the form of reaction rates ${ }^{238} \mathrm{U}$ (n, f). Signal is assumed to be the reaction rate contribution from neutron energy higher than $12.6 \mathrm{MeV}$ (unscattered) and background is the contribution in reaction rate lower than $12.6 \mathrm{MeV}$. The UVNC analysis model and channels numbering are shown in fig.3.

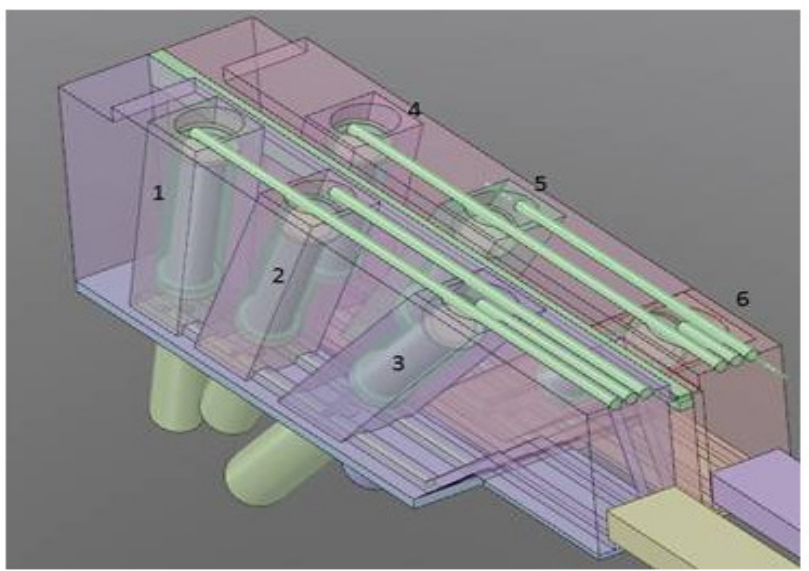

Figure 3 UVNC MCNP model and channels numbering

Results of calculations for signal/ background ratios of the FCs are presented in Table 1. The S/B ratios for the diamond detectors are factor three - four higher.

Table 1 S/B Results for UVNC FC detectors (Tungsten DM option)

\begin{tabular}{|c|c|}
\hline Channel number & S/B \\
\hline Ch\#01 & 2.2 \\
\hline Ch\#02 & 8.7 \\
\hline Ch\#03 & 2.9 \\
\hline Ch\#04 & 5.4 \\
\hline Ch\#05 & 5.3 \\
\hline Ch\#06 & 1.8 \\
\hline
\end{tabular}

As may conclude from Table 1, channels \#03 and \#06 are experiencing higher noise due to neutron background. More detailed studies to optimize the UVNC and DSM materials will be carried out to improve the S/B ratios. 


\subsubsection{Lower VNC detectors}

The LVNC's LOS pass through the Divertor Cassette and the Blanket Module \#18 structures. Collimating channels are looking from the outboard plasma region up to the plasma centre. The shielding block installed in front of the LP\#14 Diagnostic Rack hosting the Detectors Module ("nose" - red circle in fig. 4) is an important structural part because it improves the S/B ratios and decreases nuclear heating rate around detectors for about one order of magnitude.

The LVNC detectors' S/B ratios were investigated for two cases of shielding composition:

i) "Nose" is made of Tungsten and LVNC Detectors Module is made of SS316L(N)-IG;

ii) "Nose" is made of Tungsten and LVNC Detectors Module is made of mixture $10 \% \mathrm{~W}+80 \% \mathrm{~B} 4 \mathrm{C}+10 \% \mathrm{SS} 316 \mathrm{~L}(\mathrm{~N})-\mathrm{IG}$.

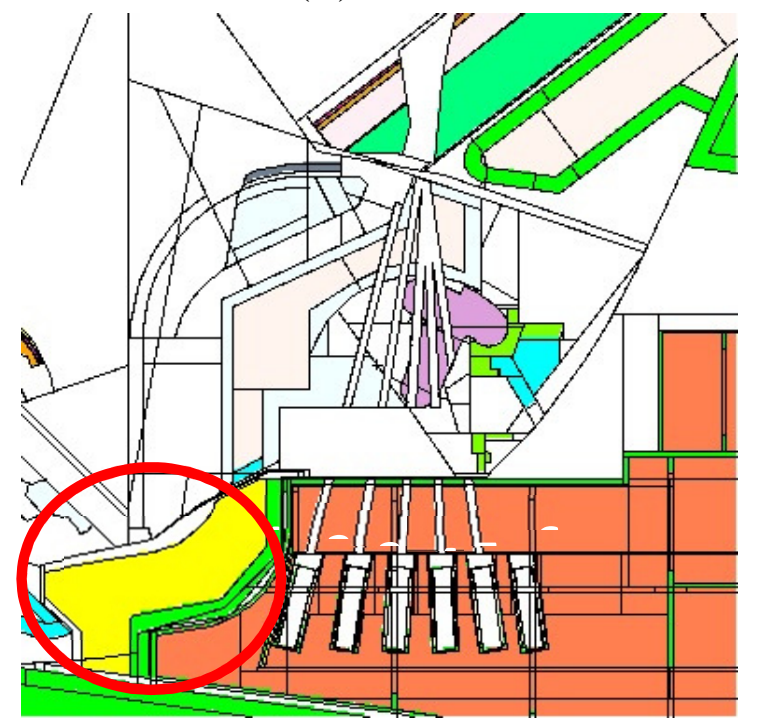

Figure 4 LVNC MCNP model

LVNC detectors' S/B ratios are shown on fig. 5. One can note that the S/B ratio has a linear behaviour with respect to the plasma view, i.e. the ratio clearly increases linearly when the direction of the neutron collimators are looking more toward the centre of the plasma. It's important to underline that the latter Detector Unit (\#6) is not equipped with collimating channel so it measures background only.

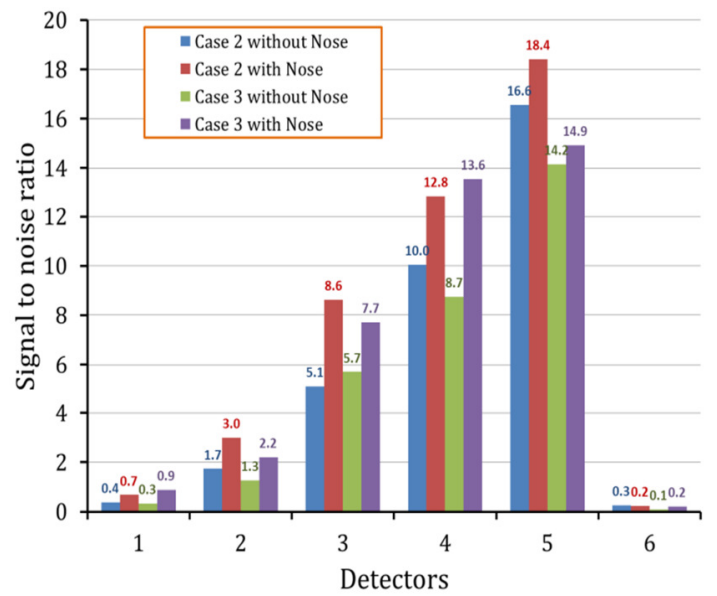

Figure 5 Results of $S / B$ assessment for LVNC FC detectors 
Like for the UVNC more detailed studies to optimize the LVNC materials will be carried out to improve the $\mathrm{S} / \mathrm{B}$ ratio.

\subsection{Dynamic range of neutron emissivity measurement}

Analysis of the dynamic range of neutron emissivity measurements for VNC neutron detectors has been based on a time resolution $1 \mathrm{~ms}$ and $10 \%$ statistical errors requirements. Due to presence of evident statistic errors minimal count rate must be at least above $10^{5} \mathrm{~Hz}$. At the same time at high count rates risk of signals pileups and following detectors saturation increases.

FCs and diamond detectors with different effective volume $V_{\text {eff }}$ are proposed for ITER VNC application. Signal count rates for these detectors are shown versus ITER DT fusion power range in fig. 6 . The estimated sensitivity of single $\mathrm{FC}$ is $10^{-3} \mathrm{~cm}^{-2}$; sensitivity of diamond detector operating with $1 \mathrm{MeV}$ registration energy threshold is $10^{-4} \mathrm{~cm}^{-2}\left(V_{\text {eff }}=1 \mathrm{~mm}^{3}\right)$ and $10^{-2}$ $\mathrm{cm}^{-2}\left(V_{\text {eff }}=100 \mathrm{~mm}^{3}\right)$ correspondingly.

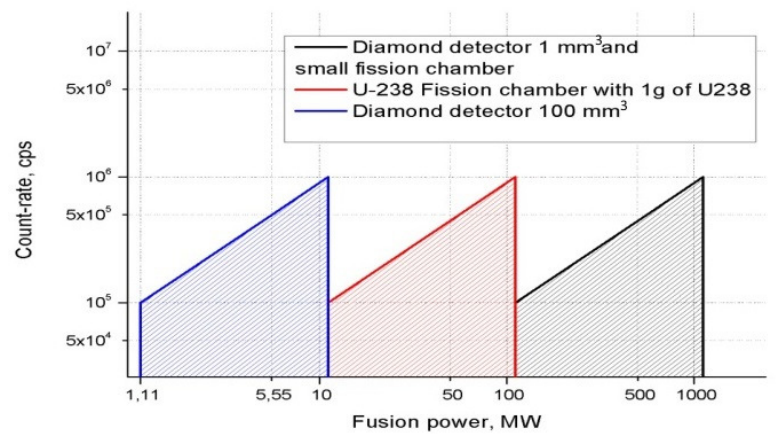

Figure 6 Signal count rate versus fusion power for different types of neutron detectors for VNC reference measurement channel (\#4, LVNC)

\subsection{Statistical error of measurement}

Statistical error for VNC detecting channels as a function of fusion power was evaluated for DT plasma operation. This analysis was performed for timing interval $1 \mathrm{~ms}$. It is assumed that detectors can operate only in counting mode (the range of rates $10^{5}-10^{6} \mathrm{~Hz}$ ) to provide the required accuracy of measurements. The statistical error for different VNC channels (DT scenario) is shown in fig. 7.

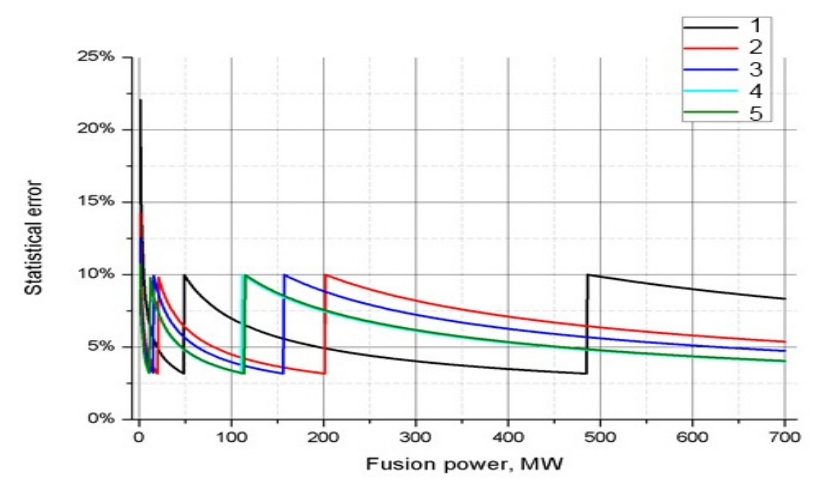

Figure 7 Measurements statistical error for different LVNC channels versus DT fusion power 
$\mathrm{R} \& \mathrm{D}$ activity has been launched on faster data acquisition system working up to $5 \mathrm{MHz}$.

VNC detectors are also functional in DD plasma operation phase but with poor temporal resolution or accuracy and a $\mathrm{R} \& \mathrm{D}$ programme is ongoing on $\mathrm{DD}$ plasma detection with higher sensitive detectors.

\section{Interfaces / Integration aspects}

LVNC has important interfaces/integrations features with the Divertor Cassette and Blanket Module \#18 - its LOS pass through these two systems. An alignment strategy is under investigation:

a) During Machine Assembly Phase I several detailed metrological campaigns will be carried out providing data on the positions of the Divertor Toroidal Rail and VV port extension and, in particular, the Divertor Radial Rails of VV port. First iteration of LVNC alignment is performed.

b) During First Plasma campaign more data will be available taking into account the information on the magnetic field lines (needed for the correct positioning of the Divertor cassettes).

c) During Machine Assembly Phase II after other metrological campaigns, customization of the LVNC Detectors and collimator modules will be carried out by means of spacers or alternative systems for the correct and final alignment which will be interrelated with the Divertor Cassette final position.

The VNC subsystems will have to be integrated with other ITER systems taking into account their requirements: Blanket, Divertor, Machine Assembly and Tooling, Remote Handling for maintenance, Cooling Water System, Service Vacuum, CODAC \& Plasma Control System, Hot Cell and Rad-waste Facility, Port Plug Test Facility and the other diagnostics tenants in UPP\#18 and in LP\#14.

\section{Conclusions}

An overview of ITER Vertical Neutron Camera (55.B2) diagnostic system dedicated to the measurement of the neutron emissivity profile and the associated challenging issues has been reported. Detailed design activity has started as well R\&D program has been launched on the various challenging issues.

\section{Acknowledgements}

The authors wish to thank: ITER Diagnostics Team, colleagues of ITER Organization and Russian Federation Domestic Agency for their support and collaboration.

Several of the MCNP geometry modelling operations were performed using the MCAM software kindly provided by the FDS Team.

\section{Disclaimer}

The views and opinions expressed herein do not necessarily reflect those of the ITER Organization. 
Concept Design and Integration Aspects of ITER Vertical Neutron Camera

\section{References}

[1] ITER Vertical Neutron Camera System Design Description Document, ITER_D_E6CM33

[2] A. Krasilnikov et al., Nuclear Fusion 45 (2005) 1503-1509

[3] M. Sasao et al., CHAPTER 9 FUSION PRODUCT DIAGNOSTICS, Fusion Science and Technology, 53(2008) 604-639

[4] M. Sasao et al., Plasma and Fusion Research, Volume 8, 2402127 (2013)

[5] Calibration of ITER neutron diagnostics, October 2013, ITER_D_NFGLTZ

[6] L. Bertalot et al, A strategy for calibrating the Neutron systems on ITER, $35^{\text {th }}$ EPS Conference on Plasma Phys. Hersonissos, ECA Vol.32D, O-2.001 (2008) 